\title{
Comparative Analysis of Hydrate Formation Pressure Applying Cubic Equations of State (EoS), Artificial Neural Network (ANN) and Adaptive Neuro-Fuzzy Inference System (ANFIS)
}

\author{
Nassim Zeinali, Mohammad Saber*, Azadeh Ameri \\ PVT Laboratory, Chemical and Petroleum Engineering Department, Sharif University of Technology, Tehran, Iran \\ E-mail: msaber@alum.sharif.edu
}

\begin{abstract}
The objective of this work is making comparison between thermodynamic models and data-driven techniques accuracy in prediction of hydrate formation pressure as a function of temperature and composition of gas mixtures. The Peng-Robinson (PR) and Patel-Teja (PT) equations of state are used for thermodynamic modeling and Artificial Neural Networks (ANNs) and Adaptive Neuro-Fuzzy Inference System (ANFIS) are used as data-driven models. The capability of each method is evaluated by comparison with the experimental data collected from literature. It is shown that there is a good agreement between thermodynamic modeling and the experimental data in most of the cases; however, the prediction relative errors are more than $10 \%$ in some cases. The data-driven models are trained and tested using a set of experimental data and their optimum structures are selected based on the prediction error of the test data set. The accuracy of ANN for prediction of hydrate formation pressure is slightly better than those of PR and PT. The prediction errors of ANFIS for all cases are less than $1 \%$ which is very promising and proves the potential of ANFIS as a capable tool for prediction of the hydrate formation pressure.
\end{abstract}

Keywords: Hydrate formation pressure; equation of state; artificial neural networks; adaptive neuro-fuzzy inference system.

\section{Introduction}

Gas hydrates are solid crystalline compounds formed under certain conditions of temperature and pressure in which gas molecules with rather modest sizes and appropriate geometries are trapped inside water cavities. The presence of water and hydrocarbon molecules in typical natural gas compositions can therefore lead to hydrate formation, which may cause serious problems in gas processing and transportation. Thus, accurate prediction of the hydrate formation condition is important to control many operational, economic and safety problems associated with the gas industry.

Several efforts have been performed to develop reliable methods of predicting gas hydrate formation condition using thermodynamic models (Chapoy et al., 2010; Ferrando et al., 2006; Marion et al., 2006; Ma et al., 2003). Some of these methods are based on a statistical thermodynamic approach developed by van der Waals and Platteeuw (Van der Waals \& Platteeuw, 1959). This approach accounts for the interactions between water molecules forming the crystal lattice and gas molecules and has been modified by many investigators (Dharmawardhana et al., 1980; John et al., 1985; Paranjpe et al., 1989; Parrish \& Prausnitz, 1972). Moreover, various empirical correlations have been presented in literatures for predicting the hydrate dissociation condition. Holder et al. have presented a correlation used for some pure gases; whereas Makogon and Kobayashi et al. have developed other general equations based on the gas gravity for hydrate forming condition of natural gases (Elgibaly \& Elkamel, 1998).

Recently, data-driven modeling methods such as Artificial Neural Networks (ANNs) are used as capable and precise mathematical techniques for prediction of phase equilibrium and PVT properties (Habiballah et al., 1996). These techniques just use the knowledge obtained from experimental input-output data of the system to predict the performance without any necessity to know the details of the related phenomena. Some recent researches have investigated the capability of ANN in prediction of hydrate formation condition (Elgibaly \& Elkamel, 1998; Mohammadi et al., 2010; Mohammadi \& Richon, 2010). Another data-driven modeling technique is the Adaptive Neuro-Fuzzy Inference System (ANFIS). Although it has been shown in some researches that ANFIS model performs better than ANN (Das et al., 2010; Esen \& Inalli, 2008), there is no published work on applying this technique for hydrate equilibrium calculations.

The objective of this work is to compare the capability of thermodynamic and data-driven modeling techniques for prediction of hydrate formation pressure. The hydrate phase equilibrium data used for training and testing the ANN and ANFIS are obtained from Sloan and Koh (Sloan \& Koh, 2008) including 722 experimental data points. These data include certain mixtures of methane, ethane, propane, ibutane, n-butane, $\mathrm{CO}_{2}$ and $\mathrm{H}_{2} \mathrm{~S}$ as gas components with temperature range of 215 to $302 \mathrm{~K}$ and pressure range of 0.05 to $62 \mathrm{MPa}$. Table 1 briefly describes the experimental data used in this work.

For thermodynamic modeling, a general phase equilibrium model based on the uniformity of fugacity of each component in all the phases is used to model the gas hydrate phase equilibria (Avlonitis, 1992; Tohidi et al., 1993). The Peng and Robinson (Peng \& Robinson, 1976) and Patel and Teja (Patel \& Teja, 1982) equations of state with the van der Waals mixing rule (Kwak \& Mansoori, 
Table 1. Some of experimental data used for training of ANN and ANFIS in this work (Sloan \& Koh, 2008).

\begin{tabular}{lccc}
\hline \multicolumn{1}{c}{ Mixture } & $\begin{array}{c}\text { Experimental data } \\
\text { number }\end{array}$ & Pressure range (MPa) & Temperature range (K) \\
\hline $\mathrm{C}_{1}, \mathrm{C}_{2}$ & 45 & $0.945-13.89$ & $214.8-293.3$ \\
$\mathrm{C}_{1}, \mathrm{C}_{3}$ & 64 & $0.245-13.89$ & $274.8-296.2$ \\
$\mathrm{C}_{1}, \mathrm{iC}_{4}$ & 77 & $0.127-62.23$ & $273.8-302.1$ \\
$\mathrm{C}_{2}, \mathrm{CO}_{2}$ & 45 & $0.565-4.081$ & $273.5-287.8$ \\
$\mathrm{C}_{1}, \mathrm{CO}_{2}, \mathrm{H}_{2} \mathrm{~S}$ & 96 & $0.582-15.707$ & $274.2-299.7$ \\
$\mathrm{C}_{1}, \mathrm{C}_{2}, \mathrm{C}_{3}, \mathrm{iC}_{4}$ & 8 & $0.92-2.67$ & $273.5-281.9$ \\
$\mathrm{C}_{1}, \mathrm{C}_{2}, \mathrm{C}_{3}, \mathrm{iC}_{4}, \mathrm{nC}_{4}$ & 5 & $0.497-13.55$ & $273.7-293.6$ \\
$\mathrm{C}_{1}, \mathrm{C}_{2}, \mathrm{C}_{3}, \mathrm{iC}_{4}, \mathrm{nC}_{4}, \mathrm{CO}_{2}$ & 16 & $0.593-3.51$ & $273.7-282$ \\
\hline
\end{tabular}

1986) are employed to calculate the fugacity in fluid phases. This approach has been proved to be a reliable tool in modeling systems containing polar and non-polar components (Avlonitis et al., 1994). For data-driven modeling, the feed-forward neural network and ANFIS are used. The authors believe that the present study is the first one applying an ANFIS for prediction of hydrate formation pressure as a function of temperature and concentration in gas mixtures.

\section{Modeling Methods}

In this section, three different modeling methods are presented. Section 2.1 describes the thermodynamic modeling and the description of ANN and ANFIS methods are stated in sections 2.2 and 2.3.

\subsection{Thermodynamic Modeling}

Generally, methods for prediction of hydrate phase equilibria are empirical correlations, charts or thermodynamic models, which are based on equality of chemical potentials of various components in different phases (Chapoy et al., 2007). Thermodynamic models are based on two different approaches to treat the VLE. One uses Equation of State (EoS) approach to calculate the fugacity of mixture components in each of the phases. The other uses EoS approach for fugacity calculation of vapor phase and employs activity coefficient and Henry's constant for modeling the aqueous phase. The latter approach is employed in this study.

Three phase equilibrium conditions $\left(\mathrm{L}_{\mathrm{w}}-\mathrm{H}-\mathrm{V}\right)$ at which hydrates form from gas and free water are considered. The $\mathrm{L}_{\mathrm{w}}-\mathrm{H}-\mathrm{V}$ line has pressure-temperature conditions of the most interest in natural gas systems, because this equilibrium normally occurs at low temperatures and high pressures (Sloan \& Koh, 2008). In fact, due to existence of water in gas sea lines and low temperature and high pressure of fluid, this three phase equilibrium is certain to take place.

\subsubsection{Hydrate Phase Modeling}

As previously mentioned, gas hydrates are crystalline compounds stabilized by inclusion of small gas molecules inside water cavities. The most common gas hydrates exist in either of two distinct structures (structures I \& II), each of which contains two different types of cavities. The chemical potentials of water in gas hydrate can be predicted from the ideal solid solution theory presented by van der Waals and Platteeuw (Van der Waals \& Platteeuw, 1959). They have assumed each cavity as a spherical cage which may contain only one gas molecule. Only gas-water interactions are allowed and it means no interactions between encaged molecules exist. According to this statistical thermodynamic theory, the chemical potential of water in a hydrate phase $\mu_{w}^{H}$ is related to that in the hypothetical empty lattice state $\mu_{w}^{\beta}$ by Eq. (1):

$$
\begin{aligned}
& \mu_{w}^{H}=\mu_{w}^{\beta}+\mathrm{R} T \sum_{i} \vartheta_{i} \ln \left(1-\sum_{K} Y_{K i}\right) \\
& i=1,2, \ldots, \mathrm{N}_{\mathrm{CAV}} \\
& K=1,2, \ldots, \mathrm{N}_{\mathrm{COMP}}
\end{aligned}
$$

where $\mathrm{R}$ is the gas constant, $T$ is the absolute temperature, $\vartheta_{i}$ is the number of cavities of type $i$ per water molecule in the unit cell, $Y_{K i}$ denotes the probability of a cavity of type $i$ ( $i$ : large cavity, small cavity) being occupied by a hydrate-forming molecule of type $K$. According to the Langmuir adsorption theory this probability is calculated from:

$Y_{k i}=\frac{C_{k i} f_{k}}{1+\sum_{j} C_{j i} f_{j}}$

$j=1,2, \ldots, \mathrm{N}_{\mathrm{COMP}}$

where $f_{k}$ is the fugacity of the gas component $K$ in the hydrate phase and $C_{K i}$ is the Langmuir adsorption constant which is specified by using statistical thermodynamics. To simplify the calculations of this parameter, it can be noticed that the Langmuir coefficients have been already evaluated by various authors. In the present model the Langmuir constants are simply considered to be temperaturedependent according to the following form derived by Munck et al. (Munck et al., 1988):

$\mathrm{C}_{\mathrm{Ki}}(T)=\frac{\mathrm{A}_{\mathrm{Ki}}}{T} \exp \left(\frac{\mathrm{B}_{\mathrm{Ki}}}{T}\right)$

The $\mathrm{A}_{\mathrm{Ki}}$ and $\mathrm{B}_{\mathrm{Ki}}$ parameters are found by adjusting them to a large number of experimental data points. The parameter values have been estimated by Munck et al. (Munck et al., 1988) which are presented in Table 2.

At equilibrium state, the chemical potential of water in hydrate phase is equal to the chemical potential of water in a coexisting phase $\alpha$ :

$\mu_{w}^{H}=\mu_{w}^{\alpha}$

Moreover, the chemical potential of water in the $\alpha$ phase may in general be written as:

$\mu_{w}^{\alpha}=\mu_{w}^{0}+\mathrm{R} T \ln \left(\frac{f_{w}^{\alpha}}{f_{w}^{0}}\right)$

where $\mu_{w}^{0}$ is the chemical potential of pure water as ice or liquid at temperature T and pressure $P, f_{w}^{\alpha}$ is the fugacity of water in the $\alpha$-phase and $f_{w}^{0}$ is the fugacity of ice or liquid water. Combination of Eq. (1) with Eq. (4) and 
Table 2. Adjusted parameters for Langmuir coefficient calculation.

\begin{tabular}{|c|c|c|c|c|c|c|c|c|}
\hline \multirow{3}{*}{ Component } & \multicolumn{4}{|c|}{ Structure I } & \multicolumn{4}{|c|}{ Structure II } \\
\hline & \multicolumn{2}{|c|}{ Small cavity } & \multicolumn{2}{|c|}{ Large cavity } & \multicolumn{2}{|c|}{ Small cavity } & \multicolumn{2}{|c|}{ Large cavity } \\
\hline & $\begin{array}{c}\mathrm{A} \times 10^{3} \\
(\mathrm{~K} / \mathrm{atm})\end{array}$ & $\begin{array}{c}\mathbf{B} \\
(\mathbf{K})\end{array}$ & $\begin{array}{c}\mathrm{A} \times 10^{3} \\
(\mathrm{~K} / \mathrm{atm})\end{array}$ & $\begin{array}{c}\mathbf{B} \\
(\mathbf{K})\end{array}$ & $\begin{array}{c}\mathrm{A} \times 10^{3} \\
(\mathrm{~K} / \mathrm{atm})\end{array}$ & $\begin{array}{c}\mathbf{B} \\
(\mathbf{K})\end{array}$ & $\begin{array}{c}\mathrm{A} \times 10^{3} \\
(\mathrm{~K} / \mathrm{atm})\end{array}$ & $\begin{array}{c}\mathbf{B} \\
(\mathbf{K}) \\
\end{array}$ \\
\hline Methane & 0.7228 & 3187 & 23.35 & 2653 & 0.2207 & 3453 & 100 & 1916 \\
\hline Ethane & 0 & 0 & 3.039 & 3861 & 0 & 0 & 240 & 2967 \\
\hline Propane & ND* & ND* & ND* & ND* & 0 & 0 & 5.455 & 4638 \\
\hline i-butane & ND* & $\mathrm{ND}^{*}$ & ND* & $\mathrm{ND}^{*}$ & 0 & 0 & 189.3 & 3800 \\
\hline n-butane & ND* & ND* & ND* & ND* & 0 & 0 & 30.51 & 3699 \\
\hline Nitrogen & 1.617 & 2905 & 6.078 & 2431 & 0.1742 & 3082 & 18 & 1728 \\
\hline $\begin{array}{l}\text { Carbon } \\
\text { dioxide }\end{array}$ & 0.2474 & 3410 & 42.46 & 2813 & 0.0845 & 3615 & 851 & 2025 \\
\hline $\begin{array}{l}\text { Hydrogen } \\
\text { Sulphide }\end{array}$ & 0.025 & 4568 & 16.34 & 3737 & 0.0298 & 4878 & 87.2 & 2633 \\
\hline
\end{tabular}

*: Not Defined (The specified components do not produce hydrate structure I)

Eq (5) gives:

$\mu_{w}^{\beta}-\mu_{w}^{0}=\mathrm{R} T \ln \left(\frac{f_{w}^{\alpha}}{f_{w}^{0}}\right)-\mathrm{R} T \sum_{i} \vartheta_{i} \ln \left(1-\sum_{K} Y_{K i}\right)$

On the other hand, based on classical thermodynamics, the chemical potential difference can be written as:

$\frac{\mu^{\beta}-\mu^{0}}{\mathrm{R} T}=\frac{\Delta \mu_{0}}{\mathrm{R} T_{0}}-\int_{T_{0}}^{T} \frac{\Delta H_{0}+\Delta C_{P}\left(T-T_{0}\right)}{\mathrm{R}^{2}} d T+\int_{P_{0}}^{P} \frac{\Delta v}{\mathrm{R} \bar{T}} d P$

$\bar{T}=\frac{T_{0}+T}{2}$

where $\Delta \mu_{0}$ is the chemical potential difference of water between the empty lattice and pure liquid water at reference condition of $\mathrm{T}_{0}=273.15 \mathrm{~K}$. Parameters of $\Delta H_{0}, \Delta C_{P}$ and $\Delta v$ stand for differences in molar enthalpy, heat capacity and volume between empty hydrate lattice and liquid water. The pressure $P_{0}$ is the water vapor pressure at $T_{0}$ which is negligible in comparison with hydrate equilibrium pressure $(P)$ and is assumed to be equal to zero absolute pressure. Based on Munck et al. (1988) assumption $\bar{T}$ is the average temperature between $T_{0}$ and the hydrate equilibrium temperature. The thermodynamic reference properties for gas hydrates for each of the two typical hydrate structures (structures I \& II) have been presented by (Munck et al., 1988) and are shown in Table 3. At hydrate equilibrium condition, the chemical potential differences which are calculated from Eq. (6) (as theoretical chemical potential difference) and Eq. (7) (as experimental chemical potential difference) should be equal.

Table 3. The thermodynamic reference properties used in Eq. (7).

\begin{tabular}{cccc}
\hline Properties & Unit & Structure I & Structure II \\
\hline$\Delta \mu_{0}$ & $\mathrm{~J} / \mathrm{mol}$ & 1264 & 883 \\
$\Delta H_{0}$ & $\mathrm{~J} / \mathrm{mol}$ & -4858 & -5201 \\
$\Delta \mathrm{v}$ & $\mathrm{m}^{3} / \mathrm{mol}$ & $4.60 \mathrm{E}-06$ & $5.00 \mathrm{E}-06$ \\
$\Delta C p$ & $\mathrm{~J} / \mathrm{mol} . \mathrm{K}$ & 39.16 & 39.16 \\
\hline
\end{tabular}

\subsubsection{Aqueous Phase Modeling}

For the aqueous phase, a Henry's law approach is used for gas components as they are at infinite dilution in water phase. Consequently, the liquid phase is supposed to be ideal and Raoult's law is applicable for solvent (water), whereas a Henry's law is used for solute (gas components).

$f_{w}^{L}=x_{w} P_{w}^{s a t} \varphi_{w}^{s a t}\left(T, P_{w}^{s a t}\right) \exp \left(\frac{v_{w}^{s a t}\left(P-P_{w}^{s a t}\right)}{\mathrm{R} T}\right)$

$f_{g}^{L}=x_{g} H_{g}^{L}(T) \exp \left(\frac{\bar{v}_{g}^{\infty}\left(P-P_{w}^{s a t}\right)}{\mathrm{R} T}\right)$

The concentration of the water $x_{w}$ is very close to unity, due to the low aqueous solubility of hydrate forming components. It can be calculated from the following equation:

$x_{w}=\frac{f_{w}^{L}}{f_{w}^{0}}=1-\sum_{i} \frac{f_{g, i}^{L}}{H_{g, i}^{L}(T) \exp \left(\frac{\bar{v}_{g, i}^{\infty}\left(P-P_{w}^{S a t}\right)}{\mathrm{R} T}\right)}$

The partial volumes used in this study are given in Table 4. For butane and hydrogen sulfide, these parameters are considered to be equal to the pure molar volumes at normal boiling points due to the fact that the partial molar volume of gases at infinite dilution in liquid solutions at $25^{\circ} \mathrm{C}$ is similar to the pure liquid volume of the solute at its normal boiling point (Poling et al., 2004).

Table 4. Partial molar volume of solute at infinite dilution.

\begin{tabular}{ccc}
\hline Component & $\overline{\boldsymbol{v}}_{\boldsymbol{g}}^{\infty}\left[\boldsymbol{m}^{\mathbf{3}} \boldsymbol{/ K \boldsymbol { m o l } ]}\right.$ & Reference \\
\hline $\mathrm{C}_{1}$ & 0.036 & {$[26]$} \\
$\mathrm{C}_{2}$ & 0.055 & {$[29]$} \\
$\mathrm{C}_{3}$ & 0.080 & {$[29]$} \\
$\mathrm{CO}_{2}$ & 0.033 & {$[25]$} \\
$\mathrm{N}_{2}$ & 0.035 & {$[29]$} \\
\hline $\mathrm{H}_{2} \mathrm{~S}$ & 0.031 & \\
$\mathrm{iC}_{4}$ & 0.092 & Calculated \\
$\mathrm{nC}_{4}$ & 0.094 & \\
\hline
\end{tabular}

The Henry's law constants for the gases can be adjusted directly from experimental results or can be taken from the literature. In this work, the Henry's constant is expressed as a function of temperature using the following equation:

$\log _{10}\left(H_{g}^{L}\right)=\mathrm{A}+\frac{\mathrm{B}}{T}+\mathrm{C} \log _{10}(T)+\mathrm{D} T$ 
where $H$ stands for the Henry's constant $(\mathrm{Pa})$ and A-D are constants adjusted for different components (Chapoy et al., 2008). Molar volume of saturated water $\left(v_{w}^{\text {sat }}\right)$ is estimated using Rackett equation (Rackett, 1970):

$v_{w}^{s a t}=v_{C} Z_{C}^{\left(1-T / T_{C}\right)^{2 / 7}}$

Vapor pressure of saturated water, $P_{w}^{s a t}$, is calculated by the following equation Danesh, (1998).

$P_{w}^{s a t}=10^{-6} \exp \left[73.649-\frac{7258.2}{T}-7.3037 \ln T+\right.$

$\left.4.1653 \times 10^{-6} T^{2}\right]$

where $P_{w}^{s a t}$ is in MPa and $T$ is in $\mathrm{K}$.

\subsubsection{Vapor Phase Modeling}

For the vapor phase modeling, the fugacity of gas components is calculated from Eq. (14).

$f_{g}^{V}(T, P, y)=\varphi_{g}^{V}(T, P, y) y_{i} P$

Fugacity coefficient calculations are dependent on the chosen equation of state. As previously stated, PengRobinson (PR) and Patel-Teja (PT) are the two selected equations employed in this work. Peng and Robinson introduced the most popular two-parameter cubic equation of state for natural gas systems. This equation assumes a fixed value for the critical compressibility factor and generally results in acceptable volumetric predictions.

Introduction of a third parameter in the equation of state relaxes the assumption of a fixed value for the critical compressibility factor and improves the prediction of the volumetric properties (Avlonitis et al., 1994). Patel and Teja introduced a third parameter in the EoS in such a way that the EoS critical compressibility factor $Z_{c}$ may be chosen freely to match the vapor pressure and saturated liquid density data. Thus, PT EoS is an improvement to the PR equation. Detailed fugacity calculations based on PT and PR equations of state are given elsewhere (Erdogmus, 2000).

\subsection{Artificial Neural Networks}

Artificial neural networks consist of the large number of computational units called neurons, connected in a massively parallel structure. The capability of learning from experimental data and simplicity of implementation are the main advantages of the ANNs over the other mathematical modeling methods. As a matter of fact, the ANN operates like a "black box" model and does not need any detailed information about the system and any explicit formulation of the mathematical equations governing the system under study (Valeh-e-Sheyda et al., 2010).

The most commonly used ANN is the feed-forward neural network which contains one input layer, one output layer and some hidden layers. The general structure of a typical feed-forward neural network is shown in Figure 1.

In each neuron, the input vector - which contains component mole fractions and temperature in this work - is multiplied by the weight parameters and then added to a scalar parameter called bias. This sum is fed to a differentiable function called transfer or activation function. Usually the sigmoid, hyperbolic tangent or linear functions are used as transfer functions in ANNs. Eventually, the neuron's output can be obtained according to the following equation (Beale \& Jackson, 1990):

$O_{j}=f\left(\sum_{i=1}^{n} w_{j i} x_{i}+b_{j}\right)$

where $O_{j}$ is the output of $\mathrm{j}^{\text {th }}$ neuron, $\mathrm{f}$ is the transfer or activation function, $b_{j}$ is the bias of $j^{\text {th }}$ neuron, $w_{j i}$ is the synaptic weight corresponding to $i^{\text {th }}$ input of $j^{\text {th }}$ neuron, $x_{i}$ is the $i^{\text {th }}$ input to $\mathrm{j}^{\text {th }}$ neuron and $\mathrm{n}$ is the number of inputs to $\mathrm{j}^{\text {th }}$ neuron.

In order to prepare a neural network for doing a special task, the synaptic weights and biases should be adjusted. This process is called training in which the connection weights are adjusted using a suitable training algorithm. The difference between the network output and the desired output is used for adjustment of network's weights and biases. Usually the Mean Square Error (MSE) is used as a criterion for training error which is defined as follows (Beale \& Jackson, 1990):

$M S E=\frac{1}{P} \sum_{p=1}^{P}\left(d_{p}-o_{p}\right)^{2}$

where $d_{p}$ and $o_{p}$ are the desired and calculated outputs for the $\mathrm{p}^{\text {th }}$ training data, respectively and $P$ is the total number of training data.

The most commonly used training algorithm is Error Back Propagation (EBP) algorithm in which the weights and biases are updated in the direction of the negative gradient of the training error as follows (Beale \& Jackson, 1990):

$w_{j i}(\mathrm{n}+1)=w_{j i}(\mathrm{n})-\eta \frac{\partial M S E}{\partial w_{j i}}$

where $n$ shows the number of iterations or epochs, and $\eta$ is the learning rate which varies between 0 and 1 .

However, EBP is the most commonly used training algorithm, but this method is often slow for practical problems. So other variants of EBP have been introduced such as Scaled Conjugate Gradient (SCG), LevenbergMarquardt (LM) and Bayesian Regulation back propagation (BR) that can converge from ten to one hundred times faster than EBP (Demuth \& Beale, 2002).

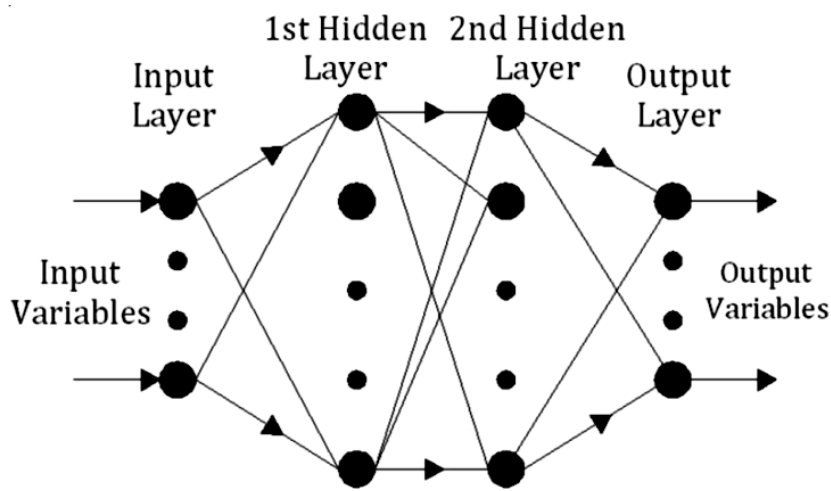

Figure 1. Structure of a typical multilayer feed forward neural network.

One of the most important problems in creating a neural network is selecting the best values of number of the hidden layers and their neurons. Also selecting the training algorithm and transfer function are equally important. 
Choosing the best structure of a neural network is often done by trial and error. It has to be mentioned that the number of neurons in the input and output layers are equal to the number of input and output variables.

After training, model validation should be done which is the process in which the input vectors which did not exist in training data set, are presented to the trained network. The capability of trained network to predict the corresponding data set is a measure of how well it has learned the patterns of training data set. The data set used for validating the trained network is called test data set.

\subsection{Adaptive Neuro-Fuzzy Inference System (ANFIS)}

The fuzzy set theory and if-then rules along with the training concept of neural networks and training algorithms form a powerful modeling method. This model applies fuzzy inference system and training algorithms of neural networks to map input variables - which are component mole fractions and temperature - to a single-variable output which is hydrate formation pressure in this work. The basic structure of a fuzzy inference system is a model that maps input characteristics to input membership functions, input membership functions to rules, rules to a set of output characteristics, output characteristics to output membership functions, and the output membership functions to a singlevalued output (Jang \& Gulley, 2002).

ANFIS structure is more complex than that of the neural networks and so there are some constraints for applying this structure to model an arbitrary input-output data set. ANFIS only supports Sugeno-type fuzzy inference system and must have the following features (Jang \& Gulley, 2002):

- Being first or zero ${ }^{\text {th }}$ order Sugeno-type system.

- Having a single output which is obtained using weighted average defuzzification.

- All output membership functions must be the same type and either linear or constant.

- Having no rule sharing. Different rules cannot share the same output membership function. In other words, the number of output membership functions must be equal to the number of rules.

To simplify the explanations, the fuzzy inference system is assumed to have two inputs ( $\mathrm{x}$ and $\mathrm{y}$ ) and one output ( $\mathrm{z}$ ). A typical fuzzy if-then rule for a first order Sugeno-type fuzzy model can be expressed as follows:

$$
\text { if } x \text { is } A \text { and } y \text { is } B \text { then } f=\mathrm{a} x+\mathrm{b} y+\mathrm{c}
$$

where $A$ and $B$ are the fuzzy sets and $a, b$, and $c$ are the parameters of fuzzy membership function of the output variable. The ANFIS' architecture with two inputs and one output is shown in Figure 2 in which a circle indicates a fixed node, whereas a square indicates an adaptive node (Jang, 1993). The output of each adaptive node depends on the parameters relating to this node. The values of these parameters are changed to minimize an error measure during the training process. For simplification, two fuzzy membership functions are assumed for each input variable as shown in Figure 2 (Jang et al., 1997).

Similar to ANN, ANFIS has a layered structure consisting of the following five layers:

Layer 1: Every node $i$ in this layer is adaptive and consists of a fuzzy set with its corresponding membership function. The output of each node in this layer is calculated by applying the fuzzy membership function on the input variables to the node:

$$
\begin{gathered}
\mathrm{O}_{1, \mathrm{i}}=\mu_{\mathrm{A}_{\mathrm{i}}}(\mathrm{x}), \text { for } \mathrm{i}=1,2 \text { and } \\
\mathrm{O}_{1, \mathrm{i}}=\mu_{\mathrm{B}_{\mathrm{i}-2}}(\mathrm{y}), \text { for } \mathrm{i}=3,4
\end{gathered}
$$

where $\mathrm{x}$ and $\mathrm{y}$ are inputs to this layer and $\mathrm{A}_{\mathrm{i}}$ and $\mathrm{B}_{\mathrm{i}}$ are linguistic values defined by fuzzy sets on the domains of $x$ and $y$, respectively. Usually the bell-shaped membership function is used. The parameters of membership functions of input variables are called antecedent or premise parameters.

Layer 2: Every node in this layer multiplies the input signals and sends the product out. For instance:

$\mathrm{O}_{2, \mathrm{i}}=\mathrm{w}_{\mathrm{i}}=\mu_{\mathrm{A}_{\mathrm{i}}}(\mathrm{x}) \times \mu_{\mathrm{B}_{\mathrm{i}}}(\mathrm{y})$, for $\mathrm{i}=1,2$

The output of each node represents the firing strength of a rule.

Layer 3: Every node in this layer calculates the ratio of the $i^{\text {th }}$ rule's firing strength to the sum of the firing strengths of all rules:

$\mathrm{O}_{3, \mathrm{i}}=\overline{\mathrm{w}}_{\mathrm{i}}=\frac{\mathrm{w}_{\mathrm{i}}}{\sum_{\mathrm{i}=1}^{2} \mathrm{w}_{\mathrm{i}}}$, for $\mathrm{i}=1,2$

For convenience, outputs of this layer will be called normalized firing strengths.

Layer 4: Every node in this layer is adaptive and multiplies the output of third layer and consequent part of the corresponding fuzzy rule:

$\mathrm{O}_{4, \mathrm{i}}=\overline{\mathrm{w}}_{\mathrm{i}} \times \mathrm{f}_{\mathrm{i}}=\overline{\mathrm{w}}_{\mathrm{i}} \times\left(\mathrm{a}_{\mathrm{i}} \mathrm{x}+\mathrm{b}_{\mathrm{i}} \mathrm{y}+\mathrm{c}_{\mathrm{i}}\right)$, for $\mathrm{i}=1,2$

The parameters in this layer $\left(a_{i}, b_{i}\right.$, and $\left.c_{i}\right)$ are referred to as the consequent parameters.

Layer 5: The last layer consists of only one node which computes the overall output as the summation of all incoming signals:

$\mathrm{O}_{5}=\sum_{\mathrm{i}=1}^{2} \overline{\mathrm{w}}_{\mathrm{i}} \mathrm{f}_{\mathrm{i}}$

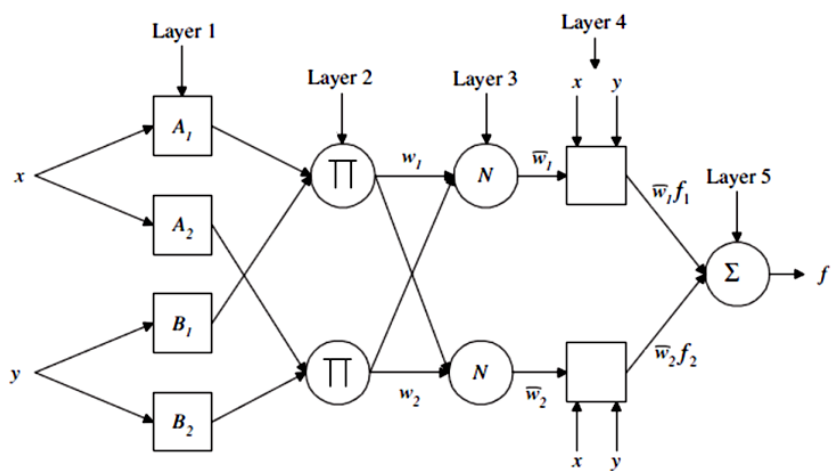

Figure 2. Simplified structure of ANFIS (Jang et al., 1997).

It can be seen that there are two adaptive layers in ANFIS architecture, namely the first layer and the fourth layer. The parameters of these layers are called antecedent (or premise) and consequent parameters, respectively. The idea of neural network training is used to train ANFIS model which means the optimization of all membership function parameters of input and output variables (e.g. antecedent and consequent parameters) in order to minimize the difference between actual output and model predicted output. Usually the Root of Mean Square Error 
(RMSE) is used as a criterion for training error which is defined as follows (Jang \& Gulley, 2002):

$R M S E=\sqrt{\frac{\sum_{p=1}^{P}\left(a_{p}-o_{p}\right)^{2}}{P}}$

where $a_{p}$ is the actual and $o_{p}$ is the predicted outputs for the $\mathrm{p}^{\text {th }}$ training data and $\mathrm{P}$ is the total number of training data.

Two different training algorithms are commonly used which are back propagation algorithm and hybrid algorithm which the latter is a combination of least squares estimation and back propagation (Jang \& Gulley, 2002).

In hybrid algorithm, the least squares method is used to optimize the consequent parameters with the assumption of constant premise parameters which is called the forward pass. When the optimal consequent parameters are found, the backward pass starts. The back-propagation algorithm (backward pass) is used to adjust the premise parameters. It has been proven that the hybrid algorithm is highly efficient in training the ANFIS (Esen \& Inalli, 2010) and so it is used in this study.

\section{Application of methods \\ 3.1 Application of EoS}

The following algorithm is implemented for equilibrium calculations in order to predict the hydrate dissociation pressure:

1. Guess initial pressure.

2. Calculation the fugacity of components in vapor phase including water vapor.

3. Calculate $x_{\mathrm{w}}$ from Eq. (10).

4. Assume initial hydrate structure (for mixtures, structure II is normally selected as the primary guess).

5. Calculate water chemical potential difference from Eq. (6) based on statistical thermodynamics.

6. Calculate water chemical potential difference from Eq. (7) based on classical thermodynamics.

7. If the calculated values in steps 5 and 6 compare favorably, then the correct dissociation pressure has been calculated for the assumed hydrate structure. Otherwise, iteration is required until the values compare within an acceptable tolerance.

8. Repeat steps 5 and 6 for the other structure considering estimated equilibrium pressure from step 7.

9. To determine the hydrate structure, two structures' chemical potential differences should be distracted as shown in Eq. (25). The hydrate structure is the one in which the chemical potential is lower. The crystal structure with the lower water chemical potential will be favored thermodynamically.

$\mu_{w}^{H, S I I}-\mu_{w}^{H, S I}=\left[(\Delta \mu)_{\text {classic }}-(\Delta \mu)_{\text {statistic }}\right]_{\text {SII }}$
$-\left[(\Delta \mu)_{\text {classic }}-(\Delta \mu)_{\text {statistic }}\right]_{S I}$

10. In case that the above difference is positive, repeat steps 1 through 7 assuming structure I to be the hydrate crystal formed.

The presented algorithm has been written in MATLAB for both PR and PT equations of state.

\subsection{Application of ANN}

In this study, a multi layer feed forward neural network has been used. Four different training algorithms including EBP with momentum, SCG, LM and BR were examined in MATLAB platform to train and validate the ANN.

The input variables of the network are temperature $[\mathrm{K}]$ and mole fractions of components in gas stream. The output variable is the pressure $[\mathrm{Pa}]$ of hydrate formation. It has to be mentioned that to achieve a better stability, the following scaling rule is applied to pressure before training (Chapoy et al., 2007):

$P_{\text {Network }}=\log \left(P_{\text {exp }}\right)$

Table 5 summarizes the basic statistics of input and output data.

Table 5. Basic statistics of input and output data.

\begin{tabular}{lcc}
\hline \multicolumn{1}{c}{ Variable } & Min & Max \\
\hline Input & 261.10 & 302.10 \\
Temperature [K] & 0 & 1 \\
Methane Mole Fraction & 0 & 0.98 \\
Ethane Mole Fraction & 0 & 1 \\
Propane Mole Fraction & 0 & 1 \\
i-Butane Mole Fraction & 0 & 0.99 \\
n-Butane Mole Fraction & 0 & 1 \\
Carbon Dioxide Mole Fraction & 0 & 0.27 \\
Hydrogen Sulfide Mole Fraction & & \\
\hline Output & 4.70 & 7.79 \\
log (Pressure [Pa]) & & \\
\hline
\end{tabular}

In order to avoid over fitting and be assured of generalization ability and predictability of the neural network, the training data set was divided into two subsets including training and test data sets. Two thirds of all experimental data were randomly selected and used for training and the rest were used for testing the network. All inputs and outputs were normalized in the interval of $[-1$, $1]$.

After preparing the training and test data sets, the network is trained with various learning algorithms, activation functions and different hidden layer neurons to obtain the optimum ANN structure. For each case, at least five runs are conducted by changing the initial weight of connections. Generally speaking, the simulation results of each run are slightly different from the others as a result of changing the initial weights.

The criterion for selection the optimum ANN structure is the Absolute Average Deviation (AAD) of test data as well as the coefficient of determination $\left(\mathrm{R}^{2}\right)$ which are defined by Eq. (27) and Eq. (28).

$A A D=\frac{1}{P} \sum_{p=1}^{P}\left|\left(\frac{o_{p}-d_{p}}{d_{p}}\right)\right| \times 100$

$R^{2}=1-\frac{\sum_{p=1}^{P}\left(d_{p}-o_{p}\right)^{2}}{\sum_{p=1}^{P}\left(o_{p}\right)^{2}}$

The optimum network structure which has been obtained by trial and error process is shown in Table 6 , and the variations of training error during training process are shown in Figure 3. As shown in Table 6, Bayesian Regulation back propagation (BR) algorithm had the optimum performance among different training algorithms. 
The concept of BR algorithm is the same as EBP although it uses second- order derivative of training error function for minimizing the training error. Furthermore, the tansig transfer function is defined by following equation (Demuth \& Beale, 2002):

$f(x)=\frac{2}{1-e^{-2 x}}-1$

Table 6. Optimum values of network parameters.

\begin{tabular}{lc}
\hline Parameters & Optimum value \\
\hline Number of Neurons & \\
Input layer & 8 \\
$1^{\text {st }}$ hidden layer & 5 \\
$2^{\text {nd }}$ hidden layer & 3 \\
Output layer & 1 \\
\hline Transfer function & tansig \\
\hline Learning rate & 0.6 \\
\hline Training algorithm & $\mathrm{BR}$ \\
\hline
\end{tabular}

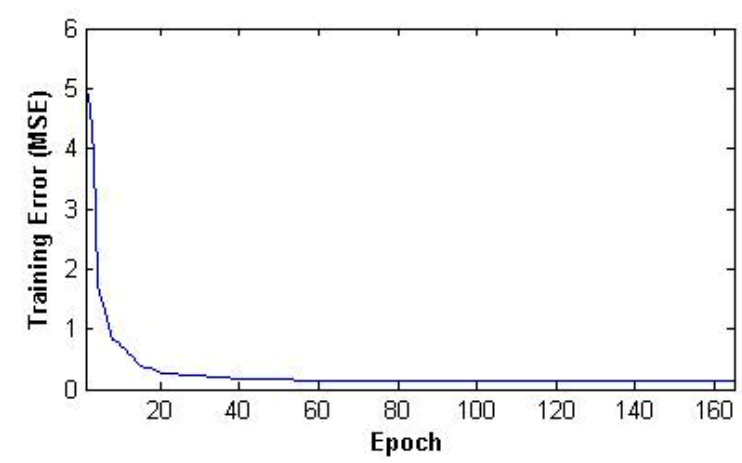

Figure 3. Training error for optimum structure of ANN.

Table 7 summarizes statistical values for training, test and all data sets. It can be seen that the MSE, $\mathrm{R}^{2}$ and AAD for all data sets are $0.146,0.992$ and $7.739 \%$, respectively. The value of $\mathrm{R}^{2}$ is near the unity which verifies that the ANN results have a good agreement with experimental data. In addition, the AAD of test data is $7.881 \%$ which is acceptable for practical applications.

Table 7. Statistical values for prediction of hydrate formation pressure (ANN model).

\begin{tabular}{lccc}
\hline Dataset & MSE & $\mathbf{R}^{\mathbf{2}}$ & AAD (\%) \\
\hline Training Data & 0.134 & 0.992 & 6.552 \\
Test Data & 0.218 & 0.990 & 7.881 \\
All Data & 0.146 & 0.992 & 7.739 \\
\hline
\end{tabular}

\subsection{Application of ANFIS}

Similar to ANN, one of the most important problems in training of ANFIS is selecting the optimum structure by trying different types and numbers of membership functions for each input variable. In the training process, the number of input membership functions varied in interval $[2,3]$ and three different types of membership functions were used including triangular-shaped (tirmf), Gaussian curve (gaussmf) and generalized bell-shaped (gbellmf) in order to obtain the optimum structure of trained ANFIS. The Fuzzy Logic Toolbox of MATLAB software has been used to implement ANFIS model in this work. The input and output variables, training and test data sets are similar to those of ANN. The AAD of test data is used as a criterion to select the optimum structure of trained ANFIS.

Table 8 shows the number of membership functions for optimum trained ANFIS structure obtained by try and error process. It should be noted that the generalized bell-shaped membership function resulted in the minimum AAD of test data and would be the best membership function for the ANFIS model. The generalized bell-shaped membership function is a three-parameter function and is defined by following equation (Jang \& Gulley, 2002):

$$
f(x, \mathrm{a}, \mathrm{b}, \mathrm{c})=\frac{1}{1+\left|\frac{x-\mathrm{c}}{\mathrm{a}}\right|^{2 \mathrm{~b}}}-1
$$

Where $a, b$, and $c$ are called antecedent or premise parameters as described in section 2.3. Figure 4 shows the graph of training error for optimum structure of ANFIS.

Table 8. Optimum number of membership functions for each input variable.

\begin{tabular}{lc}
\hline Input Variable & $\begin{array}{c}\text { Optimum number of } \\
\text { membership functions }\end{array}$ \\
\hline Temperature [K] & 3 \\
Methane Mole Fraction & 3 \\
Ethane Mole Fraction & 3 \\
Propane Mole Fraction & 3 \\
i-Butane Mole Fraction & 2 \\
n-Butane Mole Fraction & 2 \\
Carbon dioxide Mole & 2 \\
Fraction & \\
Hydrogen sulfide Mole & 2 \\
Fraction & \\
\hline
\end{tabular}

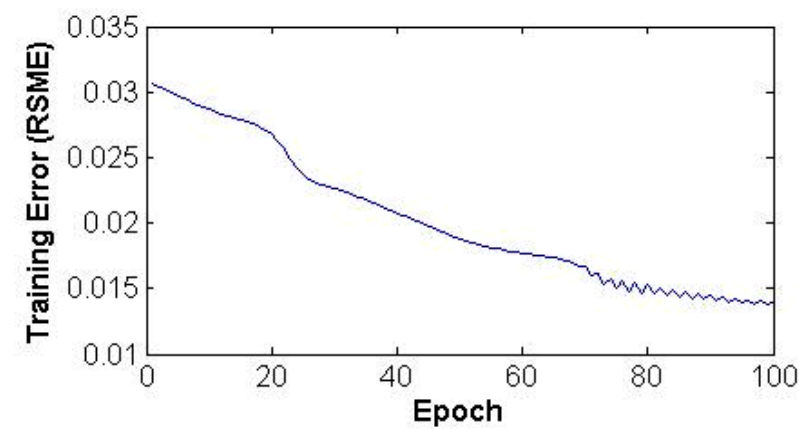

Figure 4. Training error for optimum structure of ANFIS.

The number of parameters of ANFIS in training process is given below:

- Number of input variables: 8

- Number of output variables: 1

- Number of input membership functions: 20

- Number of output membership functions: 1296

- Number of antecedent parameters: 60

- Number of consequent parameters: 3888

- Number of 'if-then' rules: 1296

- Number of nodes: 3948

- Input membership function: generalized bell-shaped

- Output membership function: linear

- Maximum number of training epochs: 100

The value of above parameters is easily calculated after selecting the optimum structure. The number of input membership functions is the summation of optimum number of membership functions for each input variable as 
mentioned in Table 8 . The number of antecedent parameters is calculated as follows:

No. of antecedent parameters $=$ No. of input membership functions * No. of parameters of each input membership function

Since in second layer of ANFIS structure, every node multiplies the input signals from each pair of input membership functions, the number of output membership functions equals to the multiplication of the number of input membership functions for each input variable. In addition, the number of consequent parameters is calculated as follows:

No. of consequent parameters $=$ No. of output membership functions * No. of parameters of each output membership function

It should be noted that the output membership function is a three-parameter linear function as described in section 2.3. The number of 'if-then' rules is the same as the number of output membership functions, and the number of the nodes is equal to the number of antecedent parameters added to the number of consequent parameters. Maximum number of training epochs is a criterion for stopping the algorithm if the training algorithm did not converge.

It has to be mentioned that if the number of input membership functions is increased, the ANFIS structure will be more complex. So, the convergence to the target error takes more iteration and the training process will be very time consuming. The training process lasted between 2 hours and 30 hours based on the number of input membership functions with a machine with a Core 2 Quad processor $2.4 \mathrm{GHz}$ and $4 \mathrm{~GB}$ RAM, whereas it took less than five minutes for neural network to be trained.

Table 9 summarizes statistical values of training, test and all data sets. As can be observed, the RMSE, $\mathrm{R}^{2}$ and AAD for all data sets are 0.0501, 0.9948 and $0.3682 \%$, respectively. The $\mathrm{R}^{2}$ of training data set is 0.999 that means ANFIS is well capable of learning from experimental data. In addition, the AAD of test data is $0.709 \%$ which is totally satisfactory. The results are very promising and it can be said that ANFIS is an appropriate technique for the prediction of hydrate formation conditions.

Table 9. Statistical values for prediction of hydrate formation pressure (ANFIS model).

\begin{tabular}{cccc}
\hline Dataset & RMSE & $\mathbf{R}^{\mathbf{2}}$ & AAD (\%) \\
\hline Training Data & 0.014 & 0.999 & 0.222 \\
Test Data & 0.085 & 0.985 & 0.709 \\
All Data & 0.050 & 0.995 & 0.368 \\
\hline
\end{tabular}

\section{Comparison of the Results}

In order to evaluate the accuracy of the thermodynamic and data-driven methods in prediction of hydrate formation pressures, the results of some three phase $\left(\mathrm{L}_{\mathrm{w}}-\mathrm{H}-\mathrm{V}\right)$ equilibrium data of different gas mixtures have been presented and compared in this section. The data used for comparison of the models are different to those used in training and testing the ANN and ANFIS. Seven cases are used for evaluating the capability of different models, which have been shown in Table 10. The results of applying different models for each case in terms of AAD are shown in Table 11, and the experimental and predicted hydrate formation pressures as a function of temperature are shown in Figure 5 through Figure 11.

The calculated ADDs for equations of state are acceptable for most of the cases. Other than cases 1 and 3 for both PT and PR and case 6 for PR, the AADs are less than $10 \%$. It should be noted that propane single hydrate formation pressure is about one order of magnitude lower than that of mixtures in cases 1 and 6 . In addition, the parameters of Langmuir reported in Table 2 are adjusted by data of single hydrate formation conditions. Therefore, the existence of propane in the mixture might have led to high ADD values in cases 1 and 6 . In case 3, high hydrate formation pressure of mixture might result in notable prediction errors since most of the equations of state could not precisely predict equilibrium at high pressure and temperature conditions. Also, polarity of $\mathrm{H}_{2} \mathrm{~S}$ might have caused deviation from proposed ideal behavior of vapor and liquid phases in equilibrium.

For cases 2 and 4 to 7, the AADs of PT is less than those of PR which means that PT has better prediction in comparison with PR. Since the equations of state are based on thermodynamic principles, they can predict the hydrate structure. Contrary to data-driven methods, EoS does not need any experimental data to predict hydrate formation conditions. However, the experimental data can be used for validating the simulation results. The comparison between ANN and EoS shows that ANN has better prediction. Other than case 3 , the prediction error of ANN is smaller than $10 \%$.

The AADs of ANFIS for all cases are less than $1 \%$ which is very encouraging and proves the potential of ANFIS as an accurate tool for prediction of hydrate formation pressure. It should be mentioned that both ANN and ANFIS are based on experimental data and for cases where not enough data is available, data-driven modeling is not applicable. Also, they are not able to predict the hydrate structure. Therefore, in spite of the fact that data-driven methods have better prediction relative to thermodynamic models, the EoS is also useful for cases in which rare experimental data is available or evaluation of hydrate structure is meant. The other point that should be noted is that the data-driven models are usually not suitable for extrapolation. So, the training data set should be selected in such a way that the whole range of input variables is adequately covered.

\section{Conclusion}

This article presents a comparative study on the prediction of hydrate formation pressure as a function of temperature and concentration in different gas mixtures using thermodynamic and data-driven methods. The accuracy of mentioned models was evaluated by comparison with several experimental cases.

Table 11. Comparison of the results.

\begin{tabular}{ccccc}
\hline Case & \multicolumn{4}{c}{ AAD $(\%)$} \\
\cline { 2 - 5 } number & PR & PT & ANN & ANFIS \\
\hline 1 & 11.67 & 12.80 & 8.98 & 0.17 \\
2 & 1.99 & 0.78 & 5.53 & 0.02 \\
3 & 11.47 & 12.93 & 10.59 & 0.53 \\
4 & 3.37 & 2.93 & 2.92 & 0.11 \\
5 & 1.89 & 1.81 & 2.68 & 0.43 \\
6 & 10.21 & 8.93 & 7.08 & 0.16 \\
7 & 4.87 & 4.19 & 4.28 & 0.53 \\
\hline
\end{tabular}


Table 10. Summary of experimental data used for model evaluation (Sloan \& Koh, 2008).

\begin{tabular}{|c|c|c|c|c|c|}
\hline $\begin{array}{c}\text { Case } \\
\text { number }\end{array}$ & Components & Reference & $\begin{array}{l}\text { Experimental } \\
\text { data number }\end{array}$ & Structure & $\Delta \mathrm{T}(\mathrm{K})$ \\
\hline 1 & $\mathrm{C}_{1}, \mathrm{C}_{2}, \mathrm{C}_{3}, \mathrm{iC}_{4}$ & Mei et al. (1998) & 8 & II & $273-278$ \\
\hline 2 & $\mathrm{C}_{1}, \mathrm{C}_{2}, \mathrm{C}_{3}, \mathrm{iC}_{4}, \mathrm{nC}_{4}, \mathrm{CO}_{2}$ & $\begin{array}{l}\text { Adisasmito and Sloan } \\
\text { (1992) }\end{array}$ & 4 & II & $273-282$ \\
\hline 3 & $\mathrm{C}_{1}, \mathrm{CO}_{2}, \mathrm{H}_{2} \mathrm{~S}$ & $\begin{array}{l}\text { Robinson and Hutton } \\
\text { (1967) }\end{array}$ & 30 & I & $279-295$ \\
\hline 4 & $\mathrm{C}_{1}, \mathrm{C}_{2}$ & $\begin{array}{l}\text { Holder and Grigoriou } \\
\text { (1980) }\end{array}$ & 14 & I & $281-288$ \\
\hline 5 & $\mathrm{C}_{1}, \mathrm{C}_{2}$ & Deaton and Frost (1946) & 24 & I, II & $274-283$ \\
\hline 6 & $\mathrm{C}_{1}, \mathrm{C}_{3}$ & Deaton and Frost (1946) & 25 & II & $274-283$ \\
\hline 7 & $\mathrm{C}_{1}, \mathrm{nC}_{4}$ & Deaton and Frost (1946) & 6 & II & $275-280$ \\
\hline
\end{tabular}

Although the prediction error was more than $10 \%$ for some cases, both PR and PT showed acceptable prediction for most of the cases. The data-driven models first were trained and tested using a set of experimental data and their optimum structures were selected according to the statistical validation results. It was concluded that the accuracy of ANN for prediction of hydrate formation pressure was slightly better than those of PR and PT. The prediction errors of ANFIS for all cases were less than $1 \%$ which is very promising and showed the potential of ANFIS as an accurate tool for prediction of hydrate formation pressure.

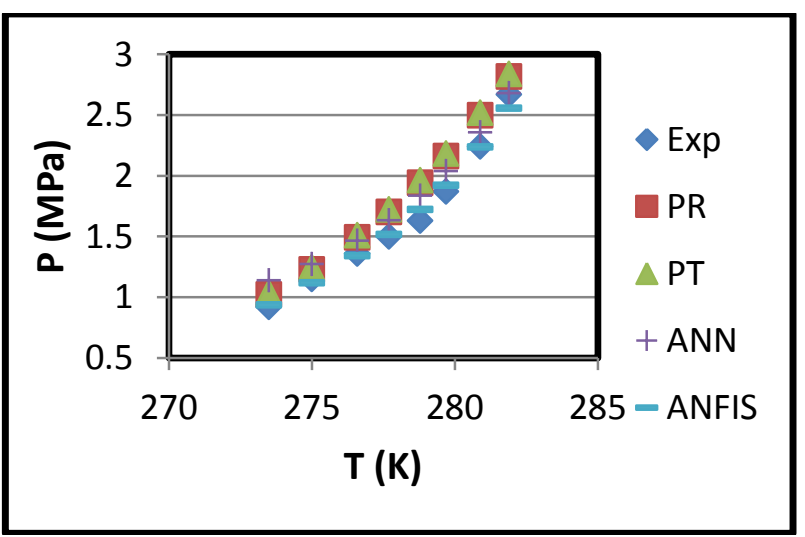

Figure 5. Experimental and predicted hydrate formation pressures as a function of temperature (case 1).

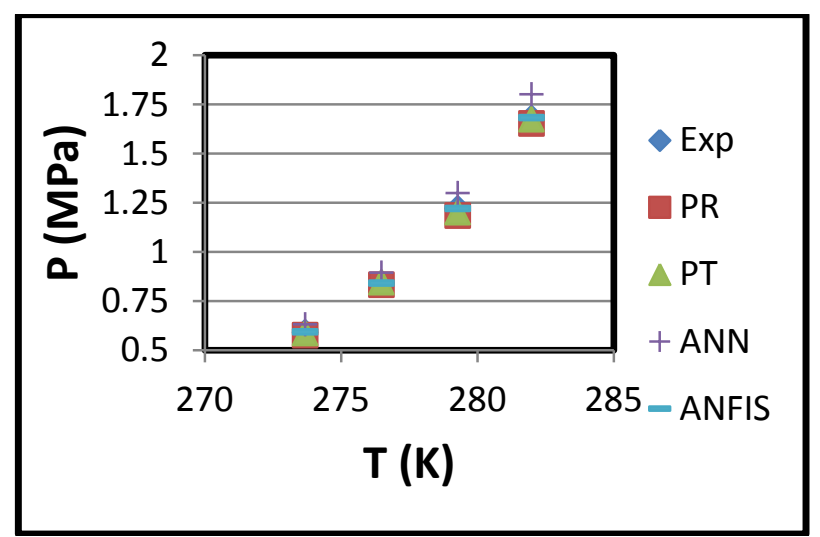

Figure 6. Experimental and predicted hydrate formation pressures as a function of temperature (case 2).

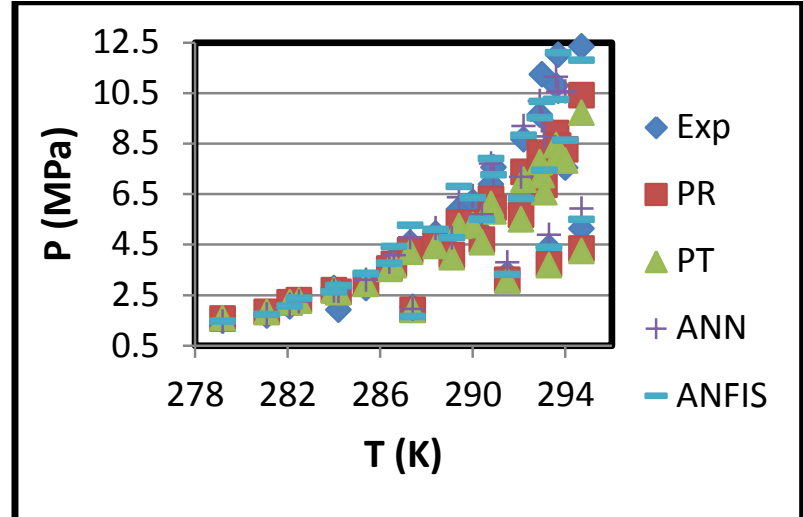

Figure 7. Experimental and predicted hydrate formation pressures as a function of temperature (case 3).

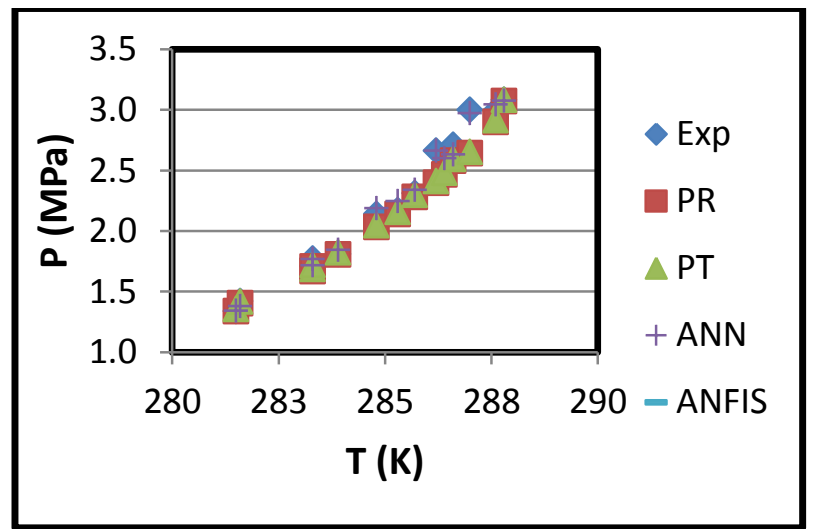

Figure 8. Experimental and predicted hydrate formation pressures as a function of temperature (case 4).

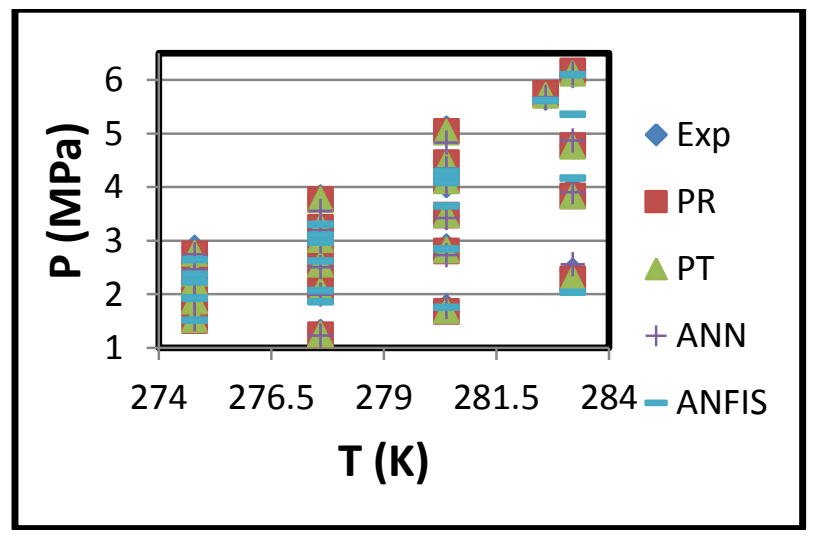

Figure 9. Experimental and predicted hydrate formation pressures as a function of temperature (case 5). 


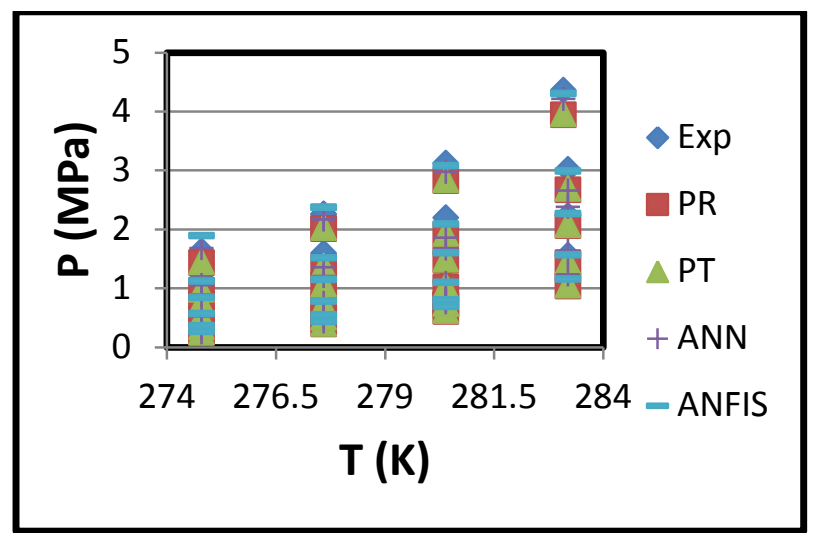

Figure 10. Experimental and predicted hydrate formation pressures as a function of temperature (case 6).

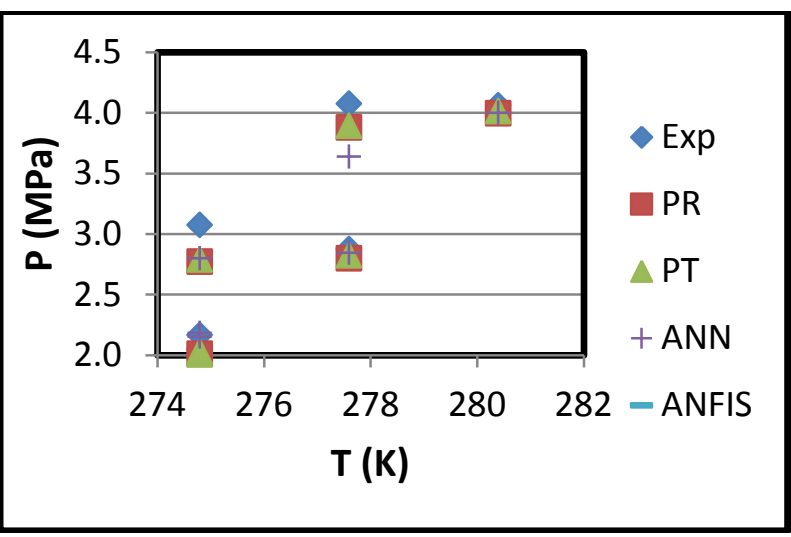

Figure 11. Experimental and predicted hydrate formation pressures as a function of temperature (case 7).

\section{Nomenclature}

$\begin{array}{ll}\text { AAD } & \text { Absolute Average Deviation } \\ \text { AD } & \text { Absolute relative Deviation } \\ \text { ANFIS } & \text { Adaptive Neuro-Fuzzy Inference System } \\ \text { ANN } & \text { Artificial Neural Network } \\ \text { SI } & \text { Hydrate structure I } \\ \text { SII } & \text { Hydrate structure II } \\ \text { VLE } & \text { Vapor Liquid Equilibrium } \\ \text { EoS } & \text { Equation of State } \\ \text { A, B , C , D } & \text { Parameters per component for henry's } \\ & \text { law correlation } \\ \text { A, B } & \text { Parameters fitted to calculate Langmuir } \\ \text { C } & \text { adsorption constant } \\ \text { H } & \text { Langmuir adsorption constant, }\left(\mathrm{MPa}^{-1}\right) \\ \text { L } & \text { Hydrate } \\ \text { P } & \text { Liquid phase } \\ \text { R } & \text { Pressure (MPa) } \\ \text { T } & \text { Universal gas constant }\left(J \cdot g m o l e^{-1} \cdot \mathrm{K}^{-1}\right) \\ \text { V } & \text { Temperature }(\mathrm{K}) \\ \text { w } & \text { Vapor phase } \\ \text { b } & \text { Neural network weights vector } \\ \mathrm{f} & \text { Neural network bias vector } \\ \text { x } & \text { Fugacity (MPa) } \\ \text { Y } & \text { Liquid phase mole fraction } \\ \text { y } & \text { Partial cavity occupation } \\ \text { Z } & \text { Vapor phase mole fraction } \\ & \text { Compressibility factor } \\ \end{array}$

\section{Greek letters}

$\mu$

$v_{i}$

$v$
Superscripts

$\mathrm{H}$

L

$\mathrm{V}$

sat

$\alpha$

$\beta$

0

$\infty$

\section{Subscripts}

C

W

i

K

0

g
Partial molar volume

Fugacity coefficient

Neural network learning rate

Hydrate phase

Liquid phase

Vapor phase

Saturated state

Coexisting phase in equilibrium with hydrate

Hypothetical empty lattice state

Reference point, pure state of water in ice or water form

Infinite dilution

Critical point

Water

Cavity type $i$

Component $K$

Reference point at $273.15^{\circ} \mathrm{K}$ and saturation vapor pressure

Solute component in aqueous phase

\section{References:}

Avlonitis, D. (1992). Thermodynamics of Gas Hydrate Equilibria. Ph.D. Thesis, Department of Petroleum Engineering, Heriot-Watt University, Edinburgh.

Avlonitis, D., Danesh, A., Todd, A.C. (1994). Prediction of VL and VLL Equilibria of Mixtures Containing Petroleum Reservoir Fluids and Methanol with a Cubic EoS. Fluid Phase Equil., 94, 181-216.

Beale, R., Jackson, T. (1990). Neural Computing: An Introduction. First ed., IOP Publishing Ltd., UK.

Chapoy, A., Coquelet, C., Richon, D. (2003). Solubility Measurement and Modeling of Methane/Water Binary System at Temperatures from 283.15 to $318.15 \mathrm{~K}$ and Pressures up to $35 \mathrm{MPa}$. Fluid Phase Equil., 214, 101117.

Chapoy, A., Haghighi, H., Burgass, R., Tohidi, B. (2010). Gas Hydrates in Low Water Content Gases: Experimental Measurements and Modelling Using the CPA Equation of State. J. Chem. Therm., 40, 10301037.

Chapoy, A., Haghighi, H., Tohidi, B. (2008). Development of a Henry's Constant Correlation and Solubility Measurements of N-Pentane, I-Pentane, Cyclopentane, N-Hexane, and Toluene in Water. J. Chem. Therm., 40, 1030-1037.

Chapoy, A., Mohammadi, A.H., Richon, D. (2007). Predicting the Hydrate Stability Zones of Natural Gases Using Artificial Neural Networks. Oil Gas Sci. Tech., $62,701-706$.

Danesh, A. (1998). PVT and Phase Behaviour of Petroleum Reservoir Fluids. Elsevier, Amsterdam.

Das, A., Maiti, J., Banerjee, R. N. (2010). Process Control Strategies for a Steel Making Furnace Using ANN with Bayesian Regularization and ANFIS. Expert Syst. Appl., 37, 1075-1085.

Demuth, H., Beale, M. (2002). Neural Network Toolbox User's Guide. The Math Works Inc. 
Dharmawardhana, P.B., Parrish, W.R., Sloan, E.D. (1980). Experimental Thermodynamic Parameters for the Prediction of Natural Gas Hydrate Dissociation Conditions. Ind. Eng. Chem. Fund., 19, 410-414.

Elgibaly, A.A., Elkamel, A.M. (1998). A New Correlation for Predicting Hydrate Formation Conditions for Various Gas Mixtures and Inhibitors. Fluid Phase Equil., 152, 23-42.

Erdogmus, M. (2000). Development of a Modified PatelTeja Equation of State. PhD. Dissertation, Pennsylvania State University, University Park, PA.

Esen, H., Inalli, M. (2010). ANN and ANFIS Models for Performance Evaluation of a Vertical Ground Source Heat Pump System. Expert Syst. Appl., 37, 8134-8147.

Ferrando, N., Lugo, R., Mougin, P. (2006). Coupling Activity Coefficient Models, Henry Constant Equations, and Equations of State to Calculate Vapor-Liquid and Solid-Liquid Equilibrium Data. Chem. Eng. Process, 45, 773-782.

Habiballah, W.A., Startzman, R.A., Barrufet, M.A. (1996). Use of Neural Networks for Prediction of Vapor Liquid Equilibrium K-Values for Light-Hydrocarbon Mixtures. SPE Reservoir Eng., 11, 121-126.

Jang, J.S.R. (1993). ANFIS: Adaptive-Network-Based Fuzzy Inference System, IEEE Trans. Syst. Man. Cybern., 23, 665-685.

Jang, J.S.R., Gulley, N. (2002). Fuzzy Logic Toolbox User's Guide. The Math Works Inc.

John, V.T., Papadopoulos, K.D., Holder, G.D. (1985). A Generalized Model for Predicting Equilibrium Conditions for Gas Hydrates. AIChE J., 31, 252-259.

Kwak, T.Y., Mansoori, G.A. (1986). Van Der Waals Mixing Rules for Cubic Equations of State, Applications for Supercritical Fluid Extraction Modeling. Chem. Eng. Sci., 41, 1303-1309.

Marion, G.M., Catling, D.C., Kargel, J.S. (2006). Modeling Gas Hydrate Equilibria in Electrolyte Solutions. Calphad, 30, 248-259.

Ma, Q.L., Chen, G.J., Guo, T.M. (2003). Modeling the Gas Hydrate Formation of Inhibitor Containing Systems. Fluid Phase Equil., 205, 291-302.

Mohammadi, A. H., Belandria, V., Richon, D. (2010). Use of an Artificial Neural Network Algorithm to Predict Hydrate Dissociation Conditions for Hydrogen + Water and Hydrogen + Tetra-N-Butyl Ammonium Bromide + Water Systems. Chem. Eng. Sci., 65, 4302-4305.

Mohammadi, A. H., Richon, D. (2010). Hydrate Phase Equilibria for Hydrogen + Water and Hydrogen + Tetra Hydro Furan + Water Systems: Predictions of Dissociation Conditions Using an Artificial Neural Network Algorithm. Chem. Eng. Sci., 65, 3352-3355.

Munck, J., Skjold-Jorgensen, S., Rasmussen, P. (1988). Computations of the Formation of Gas Hydrates. Chem. Eng. Sci., 43, 2661-2672.

Paranjpe, S.G., Patil, S.L., Kamath, V.A., Godbole, S.P. (1989). Hydrate Equilibria for Binary and Ternary Mixtures of Methane, Propane, Isobutane, and NButane: Effect of Salinity. SPE Reservoir Eng., 4, 446454.

Parrish, W.R., Prausnitz, J.M. (1972). Dissociation Pressures of Gas Hydrates Formed by Gas Mixtures. Ind. Eng. Chem. Process Des. Dev., 11, 26-35.

Patel, N.C., Teja, A.S. (1982). A New Cubic Equation of State for Fluids and Fluid Mixtures. Chem. Eng. Sci., 37, 463-473.

Peng, D.Y., Robinson, D.B. (1976). A New Two Constant Equation of State. Ind. Eng. Chem. Fund., 15, 59-64.

Poling, B.E., Prausnitz, J.P., O’Connell, J.P. (2004). The Properties of Gases and Liquids. Fifth ed., McGRAWHILL, New York.

Rackett, H.G. (1970). Equation of State for Saturated Liquids. J. Chem. Eng. Data, 15, 514-517.

Sloan, E.D., Koh, C. (2008). Clathrate Hydrates of Natural Gases. Third ed., CRC Press.

Tohidi, B., Burgass, R.W., Danesh, A., Todd, A.C. (1993). Hydrate Inhibition Effect of Produced Water, Part 1. Ethane and Propane Simple Gas Hydrates. SPE, 255264.

Valeh-e-Sheyda, P., Yaripour, F., Moradi, G., Saber, M. (2010). Application of Artificial Neural Networks for Estimation of the Reaction Rate in Methanol Dehydration. Ind. Eng. Chem. Res., 49, 4620-4626.

Van der Waals, J.H., Platteeuw, J.C. (1959). Clathrate Solutions. Adv. Chem. Phys., 11, 1-57. 